\title{
Activation of the left motor cortex during left leg movements after right central resection
}

H Gregor Wieser, Katharina Henke, Dominik Zumsteg, Ethan Taub, Yasuhiro Yonekawa, Alfred Buck

\begin{abstract}
A patient with Rasmussen's encephalitis underwent a right central resection at the age of 6 as a treatment for status epilepticus. She became seizure free, but suffered a left hemiplegia which improved so that she could walk. Because of the recurrence of seizures an enlargement of the resection to a hemispherectomy was carried out 17 years after the first operation. Various examinations, including $\mathrm{H}_{2}{ }^{15} \mathrm{O}$ PET and amytal testing, performed before this second operation indicated that a compensatory reinforcement of the ipsilateral uncrossed corticospinal and spinocortical pathways had taken place. This was confirmed postoperatively. The patient had no new sensorimotor deficits.

(F Neurol Neurosurg Psychiatry 1999;67:487-491)
\end{abstract}

Keywords: hemispherectomy; functional plasticity; $\mathrm{H}_{2}^{15} \mathrm{O}$ PET; Rasmussen's encephalitis

The prediction of the potential for recovery of functions after brain damage is of great clinical importance. One of the best examples of recovery of functions in humans is provided by patients with hemispherectomy who have regained bilateral motor functions. Considering the phenomenon of brain plasticity two main mechanisms must be considered: The "unmasking" of relatively inactive representations, and the "taking over" of function by undamaged brain tissue. ${ }^{1}$ So far it is not clear in which circumstances these two mechanisms begin to work. Several reports of patients who underwent hemispherectomy or had sustained an injury or dysfunction in the rolandic area suggest that the outcome depends on the patient's age at the time the hemisphere was damaged. ${ }^{2-6}$ One indication for hemispherectomy is Rasmussen's encephalitis which is a devastating disease causing progressive neurological deficits and intractable seizure activity in previously normal children. The disease affects one hemisphere only. The sole proved effective treatment for Rasmussen's encephalitis is hemispherectomy.

We report on a patient who had undergone a right central resection at the age of 6 because of intractable seizures due to Rasmussen's encephalitis. After surgery, she was seizure free, but displayed a left hemiplegia which, however, improved to the point that she could walk without assistance. Seventeen years later, a reoperation completing the resection to a hemispherectomy was considered because of the recurrence of seizures. This reoperation could be recommended as preoperative motor examination with $\mathrm{H}_{2}{ }^{15} \mathrm{O}$ PET and intracarotid amytal testing indicated that the patient's left leg motor functions would not further deteriorate. In fact, the results of these examinations indicated that the ipsilateral - that is, the contralesional corticospinal pathway and its collateral corticoreticulospinal pathways-had assumed control over left leg motor functions. Likewise, the somatosensory evoked potentials suggested an analogous ipsilateral compensatory reinforcement of the spinocortical pathways.

\section{Case report}

Birth and early development were normal. At the age of 5 , the patient first experienced seizures with left motor symptoms which did not respond to any of the available antiepileptic drugs. Eight months after the first seizure, she was evaluated because of drug resistant, frequent left sided tonic clonic motor and complex partial seizures. Brain CT showed atrophy of the right hemisphere. The girl exhibited a left sided paresis, mental retardation, and aggressive behaviour. Several EEGs demonstrated sharp wave activity over the right hemisphere or a delta focus over the right frontoparietal and temporal areas.

At the age of 6 , the girl developed epilepsia partialis continua with regular $1 /$ s clonic movements of the left toe and sometimes the left foot. She also exhibited a left sided motor status epilepticus involving both left limbs as well as the left side of her face. After a stereoelectroencephalographic presurgical evaluation which disclosed seizure onset at the right rolandic area, a right precentral and postcentral resection was carried out in 1980. This resection included Brodmann's area 6 on the lateral and Brodmann's areas 6 and 8 (and thereby the supplementary sensorimotor area, SSMA) on the mesial hemisphere (fig 1).

The intraoperative electrocorticographic recordings displayed a maximum of spiking in the precentral and postcentral regions including the mesial frontal and parietal areas. Histological examination of the resected specimen showed alterations compatible with Rasmussen's encephalitis. The patient became seizure free, but had a left sided hemiplegia. Yet, within months, she learned to walk unaided and was living semi-independently. With antiepileptic drug polytherapy, seizures were completely controlled until March 1989, when prolonged complex partial and brief absence-like seizures recurred. A reoperation was discussed. At the neurological examination preceding the reop- 
Op 1: $16-06-80$

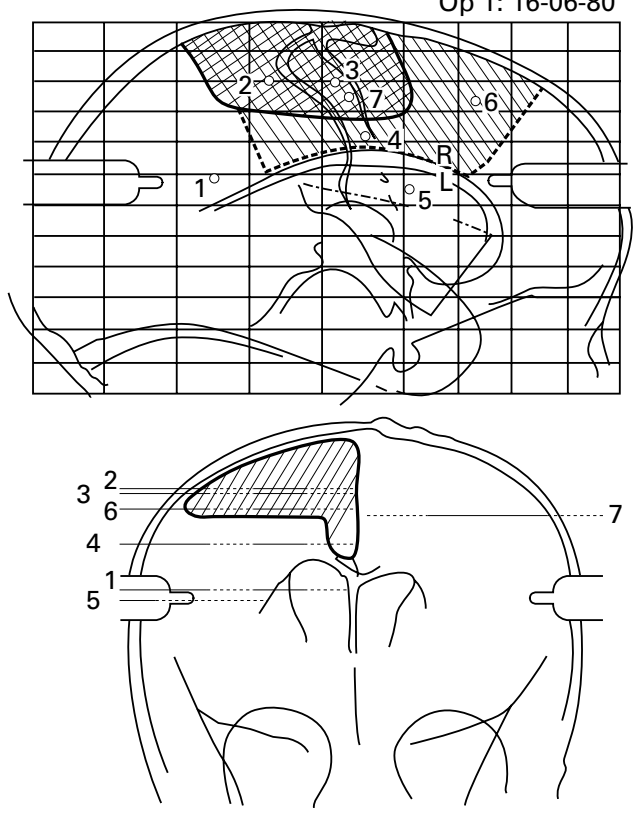

Figure 1 Schematic drawing of the results of the neuroradiological examination under stereotactic conditions (so called repérage) in the Talairach AC-PC coordinates with positioning of the seven stereotactically inserted multicontact depth electrodes and the extent of the first resection (lateral cortical aspect, double hatched; mesial aspect: hatched). Note the enlargement of the right $(R)$ ventricle system compared to the left $(L)$.

eration in October 1997, the patient showed a spastic hemiparesis on the left side involving mainly the left hand. Formal muscle testing (Medical Research Council scale) showed an essentially useless hand due to marked spasticity (3) but only moderate weakness of the biceps and triceps and shoulder muscles (4). The left leg showed a slight weakness (4). The patient was able to walk with a spastic Wernicke-Mann gait - that is, with circumduction of the left leg.

There was a minor deficit for light touch sensation and for two point discrimination on the left side, but position, vibration, temperature, and pain sensation were seemingly intact compared with the right side. Goldman perimetry showed a homonymous left sided hemianopia. At the neuropsychological examination, the patient exhibited a slightly decreased verbal recall in the Rey auditory-verbal learning test. ${ }^{7}$ The complex figure of Rey ${ }^{8}$ was copied with distortions and repetitions and was recalled later in rotation, incompletely and with misplaced details. Language functions were informally assessed, and seemed normal with the exception of orthography and arithmetic. Verbal and non-verbal fluency productions were quantitatively reduced and error prone. ${ }^{9}$ Performance in the Stroop test $\mathrm{t}^{1011}$ was good. The patient displayed psychomotor slowing, a childlike behaviour, and mood fluctuations.

Brain MRI carried out at this time showed severe damage to the right hemisphere (fig 2).

An $\mathrm{H}_{2}{ }^{15} \mathrm{O}$ PET study was performed in July 1997. PET was acquired on a whole body scanner (Advance, GE Medical Systems, Waukesha, WI, USA) in 3D mode with a $15 \mathrm{~cm}$ axial field of view. For each of 12 scans, injections of $400 \mathrm{MBq}\left[{ }^{15} \mathrm{O}\right] \mathrm{H}_{2} \mathrm{O}$ were administered as a slow bolus with a remotely controlled injection device, four during rest (=baseline), four during right leg movements, and four during left leg movements. Data were acquired over 60 seconds after the arrival of the bolus in the brain. The leg movements consisted in raising and lowering the extended leg $10 \mathrm{~cm}$ from the table and repeating these up and down movements at a frequency of one per $2 \mathrm{~s}$ during scanning $(60 \mathrm{~s})$. Before scanning, the patient was trained to carry out these movements at the right pace and to the same height with both legs. During scanning, her movements were counted and monitored by two observers ( $\mathrm{KH}$ and $\mathrm{AB}$ ) who judged them equal when carried out with either leg.

The PET scans were realigned using the least squares method implemented in SPM 95, ${ }^{12}$ then the scans were smoothed with a Gaussian filter (15 mm FWHM), and global effects such as varying injected activities were removed by dividing each voxel value by the global mean (mean of grey matter voxels). We refrained from the normalisation of the scans into the atlas of Talairach and Tournoux, ${ }^{13}$ as SPM software has difficulties spatially normalising the data of brains with large lesions. Instead, the MRI and PET were coregistered using an interactive matching too ${ }^{14}$ to allow for an accurate visual anatomical localisation of the significantly activated areas. The difference between conditions was then evaluated voxel by voxel using $t$ statistics which were subsequently transformed into normally distributed $z$ statistics. The computed comparisons were between the left leg and baseline, the right leg and baseline, and the left and right leg. Areas with large $(z>2.5, \mathrm{p}$ uncorrected $<0.006)$ resulting blood flow residuals were considered significant. The comparison of blood flow during left leg movements to rest and right leg movements to rest indicated overlapping (for each leg) significantly activated areas in the left primary motor cortex, the left insula, and superior portion of the right cerebellum (fig 3 ). Left leg movements in addition activated the left second sensory area (SSA; according to Penfield and Jasper, ${ }^{15}$ which is located on the superior bank of the fissure of Sylvius) significantly more than right leg movements and rest. Right leg movements activated the left lateral premotor cortex (Brodmann's area 6) significantly more than left leg movements and rest (fig 3). The left SSMA was activated with right as well as with left leg movements, however these activations were not quite significant compared with baseline. There were two areas of decreased blood flow during right leg movements compared to left leg movements and baseline; one of them in the left red nucleus and the other in the left prefrontal cortex (Brodmann's areas 8/9) (fig 3).

SOMATOSENSORY EVOKED POTENTIALS

Somatosensory evoked potentials (SEPs) after stimulation of the left tibial nerve resulted in a weak, distorted and very flat ipsilateral (left) cortical activation (positivity about $70-80 \mathrm{~ms}$ ). This positivity was not propagated to the con- 


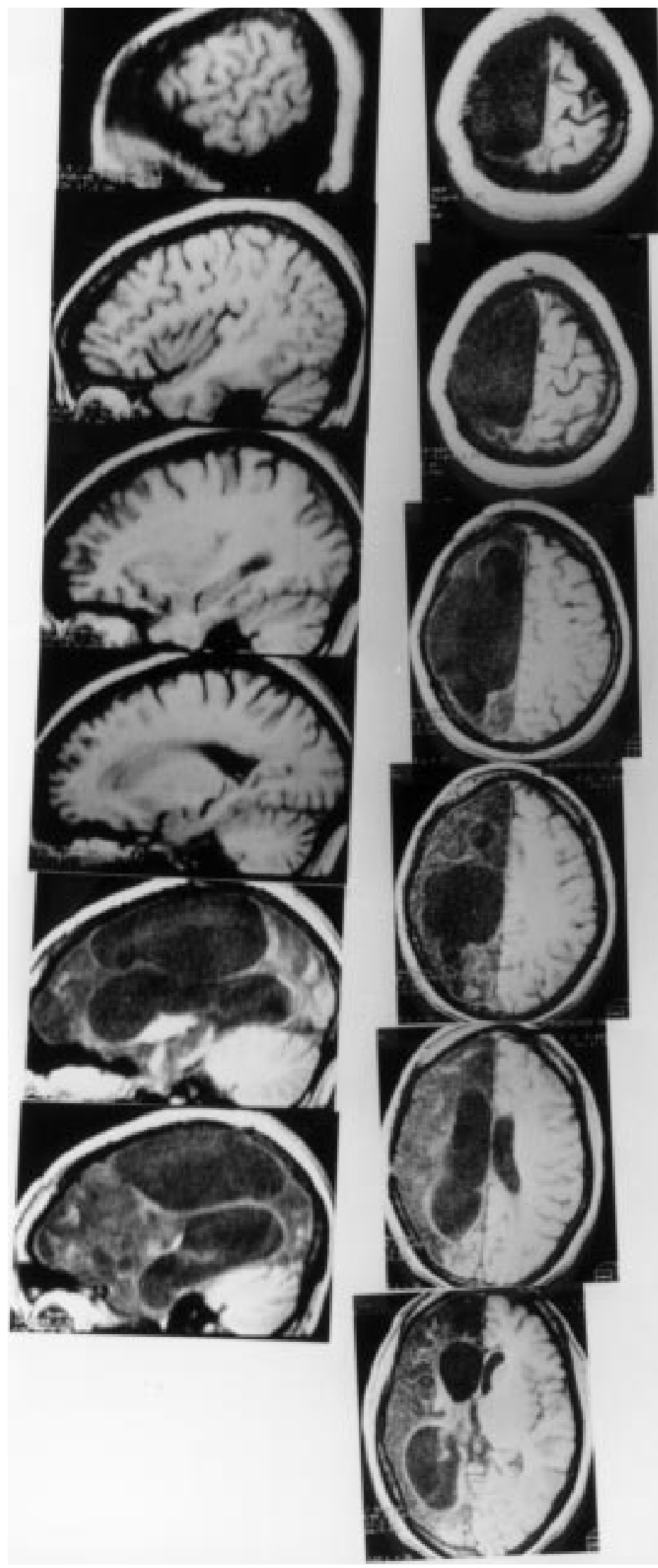

Figure 2 MRI (17 fune 1997, before the hemispherectomy) with serial sagittal and horizontal sections. Note the resection and the e vacuo enlargement of the right ventricular system, as well as the T1 hypointense signal alteration of the right brain (hyperintense in T2). There is marked atrophy of the right cerebral peduncle and to a lesser degree also of the pons besides an atrophy of the contralateral (left) cerebellum. The left cerebral hemisphere is normal. tralateral hemisphere. The SEPs after stimulation of the left median nerve showed preserved subcortical components (P15 and N18), but no cortical response (N20) over the right central area. However, there was a widely propagated positive/negative deflection with a maximum over the ipsilateral (left) somatosensory cortex. The latency of this component was in the time range of the frontal component N30 after stimulation of the normal (right) side. The SEPs after stimulation of the right side were normal.

\section{RIGHT CAROTID ANGIOGRAM}

A right carotid angiogram displayed a small calibre right internal carotid artery (ICA). The posterior cerebral artery originated directly from the ICA. The superior trunk of the right middle cerebral artery (MCA) was absent. The branches of the inferior trunk were small. A large sized right A1 gave rise to branches to the right and left paramedian regions. In the capillary phase, there was contrast in the right basal ganglia and in the left paramedian region, and markedly delayed contrast in the territory of the right MCA.

\section{RIGHT HEMISPHERE AMYTAL TEST WITH SPECT} CONTROL

A right hemisphere amytal test with SPECT control for the examination of motor and language functions was carried out. Ten $\mathrm{MBq}$ $99 \mathrm{~m}-\mathrm{HMPAO}$ and $125 \mathrm{mg}$ amytal were coinjected into the right ICA (and intravenous injection of $200 \mathrm{MBq} 99 \mathrm{~m}-\mathrm{HMPAO}$ after 20 minutes). The SPECT images showed that the amytal had inactivated the right hemisphere as well as some frontal and paramedian areas of the left hemisphere. Monitoring with EEG during the amytal test showed an initial EEG activation phenomenon with sharp waves over the right temporal area after amytal injection and then a slowing with a maximum over the left frontal and paramedian areas. On amytal injection the patient displayed an accentuation of the left facial paresis, did not respond adequately, but understood verbal commands. She showed widened pupils and nystagmoid eye movements to the right. Motor functions of legs and arms, however, remained unchanged throughout testing.

RIGHT FUNCTIONAL HEMISPHERECTOMY

A right functional hemispherectomy was carried out in October 1997 leaving the occipital polar region in situ, but transsecting the splenium of the corpus callosum. Intraoperative electrocorticography from the exposed remaining right cortex showed a severely abnormal low voltage slow activity from all right hemispheric leads. Direct electrical cortical stimulation of the exposed cortex anterior, posterior, and inferior to the former resection was done systematically. No motor response of the left limbs or the face could be elicited.

At follow up in the immediate postoperative period no change of the left sided hemiparesis but a clear reduction of spasticity was described by the neurosurgeons. At the 3 and 6 month follow up, two independent neurolo- 


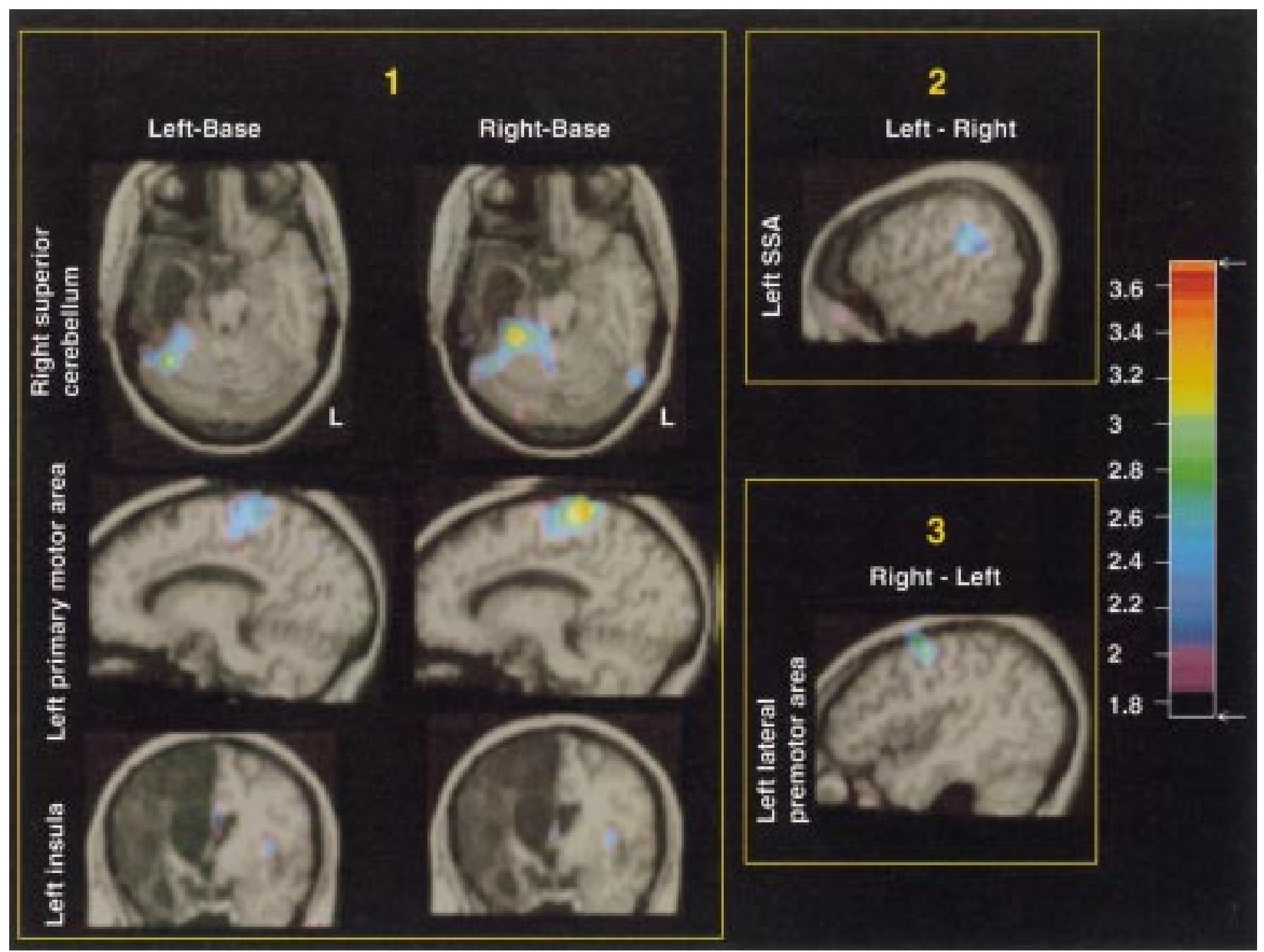

Figure 3 Significant blood flow increases as measured with $\mathrm{H}_{2}^{15} \mathrm{O}$ PET resulting from the contrasts "left leg-baseline", "right leg-baseline", and "left leg-right leg" and "right leg-left leg", fused with the patient's MRI. Base, baseline; left, left leg movements; right, right leg movements. Panel 1 displays activations which are present during movements of both legs, located in the right superior cerebellum, left primary motor area, and the left insula. Panel 2 illustrates an activation which is stronger during left than right leg movements in the left SSA (second sensory area according to Penfield and fasperis). Panel 3 illustrates an activation which is stronger during right than left leg movements in the left lateral premotor area. The bar indicates the colour coded $z$ scores starting with $z$ values at 1.8 which is below our significance threshold of 2.5.

gists found the left sided sensorimotor hemiparesis to be unchanged compared with the preoperative assessment (see preoperative examination). The neuropsychological examination 3 months after surgery disclosed a slight decrease of verbal and non-verbal memory compared with the preoperative status. According to the patient's and the parent's report, she displayed less mood fluctuations and was socially better adjusted.

\section{Discussion}

One very important factor with respect to plasticity and mechanisms of recovery is the state of brain maturation at the time of injury. Recovery after Rolandic lesions within the first years of life is greater than later in life. ${ }^{3}$ Aetiology of the lesion likewise is of great importance. Two possible mechanisms might have accounted for the recovery of motor function of the left leg in our patient: reinforcement of the ipsilateral (left) corticospinal and/or corticoreticulospinal pathways - that is, interhemispheric reorganisation, or functional taking over of left leg motor function by prefrontal or parietal cortices of the damaged right hemisphere via corticoreticulospinal pathways - that is, intrahemispheric reorganisation. The findings from several examinations carried out before the second operation suggested that the first compensatory mechanism had been at work and that intrahemispheric reorganisation had not occurred. This may be due to the nature of the patient's disease-that is, Rasmussen's encephalitis - with progressive damage of the right hemisphere. This interhemispheric reorganisation is in line with previous reports of motor recovery in patients with perinatal or early cerebral damage ${ }^{456}$ and in adult patients after hemiplegic stroke. ${ }^{16}$ The interhemispheric reorganisation involved the reallocation of the left leg motor control to the homologous primary motor region. This shift of cortical motor control to the homologous primary motor cortex is remarkable and does not confirm the finding of Müller et $a{ }^{\beta}$ of a certain resistance of cortical motor control to shift to the primary motor cortex of the contralesional hemisphere even when a lesion occurs during the first years ( $<4$ years) of life. Previous PET studies in patients with early hemispherectomy showed that motor control is reallocated more often to adjacent cortices than to the homologous primary motor cortex. ${ }^{17}$ Our PET study showed that an identical region of the left Rolandic sensorimotor cortex increased its 
blood flow during movements of the left and right leg, each compared to rest. The increase was stronger during right than left leg movements. Furthermore, no right hemisphere area was active during movements of either legthat is, only left cortical and right cerebellar regions were active during right and left leg movements.

This indicates that uncrossed pyramidal tract fibres subserved the functions of the left leg.

There is considerable variation in the estimates of the proportion of crossed and uncrossed corticospinal fibres in humans. The number of uncrossed fibres, which vary interindividually, might predict the degree of recovery of motor functions after injury to one hemisphere. Of further importance are the numerous collaterals originating from the pyramidal tract $^{18}$-namely, recurrent collaterals to the cortex, collaterals to the striatum, to the thalamus, to the red nucleus, to the reticular formation of the mesencephalon, to the pons, to the medulla oblongata, and to the cerebellum. In our patient, right and left leg movements led to an equally strong activation of the right cerebellum.

Corresponding to the finding that the SSMA on the mesial aspect of the frontal lobe contributes much to the ipsilateral innervation, ${ }^{19} 20^{21}$ we found activation of the left SSMA during left (and right) leg movements. With regard to the preservation of the sensory function of the left side, the ipsilateral cortical activation in the SEP after stimulation of the left leg is intriguing, as it resembled the second contralateral positivity after stimulation of the normal side, yet it was lacking the propagation to the other hemisphere. A generator in the convexity of the ipsilateral (left) hemisphere is therefore assumed. The widely propagated positive/negative deflection after stimulation of the left arm was similar to the frontal N30 component after stimulation of the right median nerve with respect to configuration and latency. It is suggested that the N30 component is generated in the SSMA ${ }^{22} 23$ and that it is relatively preserved after resection of the postcentral gyrus. ${ }^{24}$ This positive/negative deflection might therefore reflect a compensatory ipsilateral activation of the SSMA after sensory stimulation of the paretic left hand.

In summary, we present PET and SEP evidence that both the ipsilateral corticospinal tract with its collaterals and the ipsilateral spinocortical tract were reinforced. These findings were validated by the results of the preoperative amytal test, the intraoperative electrocorticography and electrical brain stimulation and, most importantly, by the good postopera- tive functional outcome: the patient had not had an additional sensorimotor deficit.

We thank our colleagues A Aguzzi, A Gooss, M Hajek, S Müller, M Regard, B Schuknecht, R Schiess, and B Weber for their help in the course of the assessment of this patient. This research was in the course of the assessment of this patient. This research was supported by

1 Bach-y-Rita P. Brain plasticity as a basis for recovery of function in humans. Neuropsychologia 1990;28:547-54.

2 Benecke R, Meyer BU, Freund HJ. Reorganisation of descending motor pathways in patients after hemispherectomy and severe hemispheric lesions demonstrated by tomy and severe hemispheric lesions demonstrated by 26 .

3 Müller R-A, Rothermel RD, Behen ME, et al. Plasticity of motor organization in children and adults. Neuroreport 1997;8:3103-8

4 Cao Y, Vikingstad EM, Huttenlocher PR, et al. Functional magnetic resonance studies of the reorganization of the human hand sensorimotor area after unilateral brain injury in the perinatal period. Proc Natl Acad Sci USA 1994;91:9612-6.

5 Nirkko AC, Rösler KM, Ozdoba C, et al. Human cortical plasticity: functional recovery with mirror movements. Neurology 1997;48:1090-3.

6 Sabatini U, Toni D, Pantone P, et al. Motor recovery after early brain damage. A case of brain plasticity. Stroke 1994; 25:514-7.

7 Rey A. L'examen clinique en psychologie. Paris: Presses universitaires de France, 1958

8 Rey A. Test de copie et de reproduction de mémoire de figures géométriques complexes. Paris: Ed Centre Psychol Appl, 1959.

9 Regard M, Strauss E, Knapp P. Children's production on verbal and nonverbal fluency tasks. Percept Mot Skills 1982; 55:839-44.

10 Stroop JR. Studies of interference in serial verbal reactions. 7 Exp Psychol 1935;18:643-62.

11 Perret E. The left frontal lobe of man and the suppression of habitual responses in verbal categorical behavior. Neuropsychologia 1974;12:323-30.

12 Frackowiak RSJ, Friston KJ. Functional neuroanatomy of the human brain: positron emission tomography: a new neuroanatomical technique. f Anat 1994;184:211-25.

13 Talairach J, Tournoux P. Co-planar stereotaxic atlas of the human brain. Stuttgart: Thieme, 1988.

14 Pietrzyk U, Herholz K, Fink G, et al. An interactive technique for three-dimensional image registration: validation for PET, SPECT, MRI and CT brain studies. $\mathcal{F} \mathrm{Nucl}$ Med 1994;35:2011-8.

15 Penfield W, Jasper H. Epilepsy and the functional anatomy of the human brain. London: Churchill Livingstone, 1954.

16 Chollet F, DiPiero V, Wise RJS, et al. The functional anatomy of motor recovery after stroke in humans: a study with positron emission tomography. Ann Neurol 1991;29: 63-71.

17 Müller RA, Chugani HT Muzik O, et al. Brain organization of motor and language functions following hemispherectomy: a [(15)O]-water positron emission tomhemispherectomy: a $[(15) \mathrm{O}]$-water positron

18 Nyberg-Hansen R, Rinvik E. Some comments on the pyramidal tract, with special reference to its individual variations in man. Acta Neurol Scand 1963;39:1-30.

19 Bates JAV. Stimulation of the medial surface of the human cerebral hemisphere after hemispherectomy. Brain 1953; 76:405-47.

20 Lüders HO. Supplementary sensorimotor area. Advances in neurology. Vol 10. Philadelphia: Lippincott-Raven, 1996: 512.

21 Muller F, Kunesch B, Binkofski F, et al. Residual sensorimotor functions in a patient after right-sided hemispherectomy. Neuropsychologia 1991;29:125-45.

22 Desmedt JE, Bourguet M. Color imaging of parietal and frontal somatosensory evoked potential fields evoked by stimulation of median or posterior tibial nerve in man. Electroencephalogr Clin Neurophysiol 1985;62:1-17.

23 Maugière F, Desmedt JE. Focal capsular vascular lesions can selectively deafferent the prerolandic or the parietal cortex: somatosensory evoked potentials evidence. Ann Neurol 1991;30:71-5.

24 Slimp JC, Tamas LB, Stolov WC, et al. Somatosensory evoked potentials after removal of somatosensory cortex in man. Electroencephalogr Clin Neurophysiol 1986;65:111-7. 